\title{
Study of patients' dehumanization in a hospital setting
}

\author{
Dimitra Lekka ${ }^{1}$, Maria Sakalaki ${ }^{2}$ \\ ${ }^{1}$ Psychiatric clinic Sotiria Chest Hospital, MSc, PhD candidate University of Social and Political Sciences, Department of \\ Psychology, Athens, Greece \\ ${ }^{2}$ Department of Psychology Panteion University of Social and Political Sciences, Athens, Greece
}

Background: Dehumanization is historically a well-known phenomenon and it has been studied a lot in terms of intergroup relationships. Dehumanization is defined as a denial of humanness to others. There are two forms dehumanization, animalistic, which denies characteristics that distinguish humans from animals, and mechanistic, which denies capacities that separate people from machinery. There is a lack of research on dehumanization of patients among Greek healthcare professionals.

Purpose: This study aims to explore and compare the extent and the forms of patients' dehumanization among Greek health professionals, mental health professionals and the general population.

Methods: A translated version of Haslam's questionnaire addressing both types of dehumanization was distributed to health and mental health professionals in two public hospital settings and the general population. Moreover, the Greek versions of Fraley's et al. Adult attachment patterns questionnaire and Deci \& Ryan self-determination questionnaire were distributed to all three groups.

Results: The sample included 353 participants (103 males, 250 females, age 18-60 years). 135 of them were mental health professionals (physicians and nurses), 134 were health professionals (physicians and nurses of pathological and surgery departments) and 84 were from the general population. The results showed that participants scored similarly on mechanistic $(\mathrm{M}=5.00, \mathrm{SD}=1.069)$ and animalistic dehumanization $(\mathrm{M}=5.10$, $\mathrm{SD}=1.267)$. One way ANOVA analysis revealed that health care professionals showed significantly higher mechanistic dehumanization $(\mathrm{M}=5.12, \mathrm{SD}=1.049)$ as compared to the general population $(\mathrm{M}=4.75$, $\mathrm{SD}=1.023), \mathrm{F}(2,350)=3.290, \mathrm{p}=.038$. There was no statistically significant difference among the other groups in terms of both forms of dehumanization. Regression analysis showed that the dimension of avoidance in adult attachment significantly predicted mechanistic dehumanization, accounting for $58 \%$ of the variance in mechanistic dehumanization $(\beta=.149, \mathrm{t}=2.453, \mathrm{p}=.015)$, as well as animalistic dehumanization, accounting for $54 \%$ of the variance in animalistic dehumanization $(\beta=.185, t=2.546, p=.011)$. The level of education was also a significant predictor of both mechanistic dehumanization $(\beta=.265, t=3.500, p=.001)$ and animalistic dehumanization $(\beta=.204, \mathrm{t}=2.241, \mathrm{p}=.026)$ Orientation of control was a significant predictor of animalistic dehumanization $(\beta=.195, \mathrm{t}=1.998, \mathrm{p}=.047)$.

Conclusion: The findings show high rates of dehumanization among all three groups. The design and implementation of relevant strategies may decrease or prevent this phenomenon.

Key words: patients' dehumanization, mechanistic and animalistic dehumanization 